\title{
Übersichten
}

Zbl Arbeitsmed $2020 \cdot 70: 240-246$ https://doi.org/10.1007/s40664-020-00387-6 Online publiziert: 14. Februar 2020

(c) Der/die Autor(en) 2020

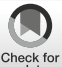

\author{
S. Sammito ${ }^{1,2} \cdot$ F. Heblich ${ }^{3,4} \cdot$ N. Güttler ${ }^{5}$ \\ 'Fachgruppe I 3 Foschung \& Erprobung, Zentrum für Luft- und Raumfahrtmedizin der Luftwaffe, Köln, \\ Deutschland \\ ${ }^{2}$ Bereich Arbeitsmedizin der Medizinischen Fakultät, Otto-von-Guericke Universität Magdeburg, \\ Magdeburg, Deutschland \\ ${ }^{3}$ Schiffsarztlehrgang GbR, Kiel, Deutschland \\ ${ }^{4}$ Betriebsärztlicher Dienst, Christian-Albrechts-Universität zu Kiel, Kiel, Deutschland \\ ${ }^{5}$ Klinische Flugmedizin, Zentrum für Luft- und Raumfahrtmedizin der Luftwaffe, Fürstenfeldbruck, \\ Deutschland
}

\section{Die Fahrradergometrie in der arbeitsmedizinischen Vorsorge}

der Novellierung der ArbMedVV individuell an den Probanden anzupassen, „Kochrezepte“ sind weder mit den rechtlichen Vorgaben, noch mit den aktuellen wissenschaftlichen Empfehlungen vereinbar. So stellt die (Fahrrad-)Ergometrie gerade für die oben aufgeführten Berufsfelder eine häufig diskutierte Untersuchungsmethode zur Feststellung der körperlichen Leistungsfähigkeit und im Rahmen der Diagnostik kardialer Erkrankungen dar.

\section{Methode}

Auf Basis einer selektiven Literaturrecherche unter Einbeziehung von Empfehlungen aus Leitlinien und Lehrbüchern zur Fahrradergometrie ist es das Ziel dieses Übersichtsartikels, dass der Leser im Anschluss die Möglichkeiten, aber auch die Grenzen der Fahrradergometrie für den Einsatz im Rahmen der arbeitsmedizinischen Vorsorge und Eignungsuntersuchung kennt.

\section{Wissenschaftliche Empfehlungen}

National und international existieren Empfehlungen der jeweiligen Fachgesellschaften zur Durchführung der Ergometrie, welche zumeist von den kardiologischen bzw. sportmedizinischen Fachgesellschaften publiziert wurden (u. a. [5-8]). Gerade für den deutschsprachigen Raum datieren diese jedoch auf die Jahre 2000 (Deutsche Gesellschaft für Kardiologie [7]) bzw. 2006 (Österreichische Kardiologische Gesellschaft [8]) und wurden seitdem nicht mehr aktualisiert. Darüber hinaus besteht für den Arbeits- und Betriebsmediziner die Möglichkeit, in entsprechenden Lehrbüchern, Übersichtsartikeln und Positionspapieren weitergehende Empfehlungen und Informationen zum Einsatz der (Spiro-)Ergometrie zu finden [9, 25-27].

Es gilt beim Einsatz der Fahrradergometrie $\mathrm{zu}$ berücksichtigen, dass die insbesondere von klinischen Fachgebieten herausgegebenen Empfehlungen den Einsatz dieser Untersuchungsmethode zur Prognoseabschätzung sowie zur Therapie- und Interventionsüberwachung im Rahmen einer Krankheit betrachten, ferner zur Diagnostik latenter Erkrankungen und im Besonderen im sport- bzw. rehabilitationsmedizinischen Kontext [10]. Darüber hinaus wird diese, zumeist eher sekundär, zur Einschätzung der Belastbarkeit bzw. zur Leistungsdiagnostik mit Trainingsberatung, -kontrolle und -steuerung eingesetzt [10].

\section{Definition}

Die quantitative Messung und Beurteilung der körperlichen Leistungsfähigkeit und Belastbarkeit von Gesunden und Kranken wird als Ergometrie verstanden [9]. Die Ergometrie erfolgt mit einer definierten Belastung, soll reproduzierbar, dosierbar, vergleichbar und objektiv sein. Die Ergometrie setzt, wie eine vergleich- 
Tab. 1 Kontraindikationen zur Ergometrie. (Mod. nach $[9,27]$ )

Kontraindikation

Absolut Akuter Myokardinfarkt

Instabile Angina pectoris

Herzrhythmusstörungen mit Symptomatik und/oder eingeschränkter Hämodynamik

Symptomatische schwere Aortenklappenstenose

Dekompensierte Herzinsuffizienz

Akute Lungenembolie

Akute entzündliche Herzerkrankungen

Akute Aortendissektion

Relativ Hauptstammstenose

Klappenerkrankungen mäßigen Schweregrades

Bekannte Elektrolytstörungen

Arterielle Hypertonie (systolisch >200 mm Hg, diastolisch $>110 \mathrm{~mm} \mathrm{Hg}$ )

Tachyarrhythmie oder Bradyarrhythmie

Hypertrophisch-obstruktive Kardiomyopathie und andere Formen der Ausflussbahnobstruktion

Höhergradige AV-Blockierungen

Physische und/oder psychische Beeinträchtigungen

Andere Arrhythmien

bare Labormethode, ein standardisiertes Vorgehen mit Qualitätskontrolle voraus. In der Arbeitsmedizin haben sich fahrradergometrische Untersuchungen etabliert, es existieren darüber hinaus aber grundsätzlich eine beliebige Anzahl weiterer Möglichkeiten, die den aufgeführten Qualitätskriterien entsprechen und je nach Anwendungsfall des jeweiligen Fachgebiets bzw. der Fragestellung auch geeigneter sein können (z.B. Kletterstufe, Laufbandergometrie, Armkurbelergometer, Schwimmergometrie etc.). Darüber hinaus werden im internationalen Vergleich erschwerend unterschiedliche Methoden eingesetzt. So ist beispielhaft die Laufbandergometrie mittels entsprechender Protokolle in den USA deutlich etablierter als die Fahrradergometrie [11].

\section{Voruntersuchung}

Vor der Durchführung einer Fahrradergometrie gilt es, eine ausführliche Anamnese beim Probanden durchzuführen, welche insbesondere die unten aufgeführten Kontraindikationen ausschließt und die aktuelle Medikation umfasst sowie den Probanden über Ablauf und medizinischer Absicht der Ergometrie aufzuklären. Die Aufklärung sollte schriftlich erfolgen $[9,27]$. Vor Beginn der eigentlichen Belastung ist der Ruhe-Blutdruck zu bestimmen sowie ein RuheEKG durchzuführen und ärztlich zu beurteilen $[9,27]$. Ferner sollte eine klini- sche Untersuchung mit mindestens Auskultation des Herzens und der Lunge erfolgen $[9,27]$. Bei auffälligen klinischen oder elektrokardiographischen Befunden sollte vor einem Belastungstest eine weitergehende kardiologische $\mathrm{Ab}$ klärung (u.a. mittels echokardiographischer Untersuchung) vorausgehen [27]. Die Probanden sollten einen empfohlenen Mindestabstand von $2 \mathrm{~h}$ zur letzten Mahlzeit und zu schweren körperlichen Belastungen einhalten und $12 \mathrm{~h}$ vor der Ergometrie keinen Alkohol oder Nikotin konsumiert haben $[9,27]$. Durchgeführte (auch $>2 \mathrm{~h}$ ) schwere körperliche Tätigkeiten beeinträchtigen die Aussagekraft derErgometriehinsichtlich der absoluten maximalen Leistungsfähigkeit, da diese grundsätzlich nur im ausgeruhten und erholten Zustand erreicht wird.

Es bestehen relative und absolute Kontraindikationen für die Durchführung einer Belastungsuntersuchung, die vor der Ergometrie ausgeschlossen werden sollten (- Tab. 1). Gerade im arbeitsmedizinischen Bereich als elektive Maßnahme unter leistungsdiagnostischer Fragestellung und bei ggf. nur geringer regelmäßiger Erfahrung in der Durchführung der Untersuchung sollte auch an die relativen Kontraindikationen ein strenger Maßstab angesetzt werden. Ferner muss der Durchführende zwingend Erfahrungen in der notfallmedizinischen Behandlung möglicher Komplikationen einschließlich des Herz-Kreislauf-Stillstandes inklusive der apparativen und medikamentösen Ausstattung besitzen [10].

\section{Untersuchungsprogramm}

Früher zumeist als Untersuchungsprotokoll bezeichnet, hat sich der Begriff Untersuchungsprogramm inzwischen durchgesetzt. Hierbei sollten bei einem Stufenprogramm folgende Aspekte berücksichtigt werden [9, 27]:

- die Gesamtdauer der Belastung sollte zwischen 10 und 12 min umfassen, längere Belastungsphasen bergen das Risiko einer zunehmenden Überhitzung des Probanden, kürzere sind nur eingeschränkt auswertbar,

- im Rahmen arbeitsmedizinischer Untersuchungen sollte mit einer Anfangsbelastung von $50 \mathrm{~W}$ gestartet werden (in Abhängigkeit von der Sportanamnese ggf. auch höher, bis max. $100 \mathrm{~W}$ ),

- die Belastung sollte entweder stufenweise alle $2 \mathrm{~min}$ (Stufenbelastung) oder kontinuierlich (Rampenbelastung) gesteigert werden,

- die Steigerung sollte in der Regel $25 \mathrm{~W}$ pro $2 \mathrm{~min}$ Stufe bzw. $25 \mathrm{~W}$ pro $2 \mathrm{~min}$ in einem Rampenprotokoll bis zur Ausbelastung betragen,

- der Belastung wird eine 5-minütige Vorphase ohne Belastung vorgeschaltet, als Ruhewerte werden die Messungen der letzten $3 \mathrm{~min}$ vor Belastungsbeginn herangezogen und

- nach Belastungsabbruch ist eine mindestes 6-minütige Nachbeobachtungsphase mit weiterhin kontinuierlicher EKG-Registrierung und regelmäßiger Messung von Blutdruck und Herzschlagfrequenz einzuplanen.

\section{Stufen- vs. Rampentest}

Grundsätzlich sind stufen- von rampenförmigen Belastungen im Rahmen der Fahrradergometrie zu unterscheiden. Während bei ersteren eine Belastungsstufe für eine gewisse Zeit (in der Regel $2 \mathrm{~min}$ ) beibehalten wird (z. B. $50 \mathrm{~W}$ als 1. Stufe) und nach Ablauf dieser Zeit eine plötzliche Steigerung um eine vorher definierte Belastung (z.B. $+25 \mathrm{~W}$ ) erfolgt, welche wiederum für eine gewisse Zeit 


\section{S. Sammito · F. Heblich $\cdot$ N. Güttler}

\section{Die Fahrradergometrie in der arbeitsmedizinischen Vorsorge}

\section{Zusammenfassung}

Eine ausreichend hohe körperliche Leistungsfähigkeit ist für viele Berufsgruppen von elementarer Bedeutung für die Bewältigung der täglichen Arbeitsanforderungen (u.a. Feuerwehr, Polizei, Militär, aber auch Beschäftigte im Baugewerbe, Bergbau und Landwirtschaft). Für die Überprüfung der körperlichen Leistungsfähigkeit steht dem Arbeits- und Betriebsmediziner die (Fahrrad-)Ergometrie als standardisierte, unter Praxisbedingungen durchführbare Untersuchung zur Verfügung. Unter Berücksichtigung der nationalen und internationalen Leitlinien und aktueller wissenschaftlicher Literatur werden die für den Arbeitsmediziner notwendigen Kenntnisse, Voraussetzungen, Indikationen und Kontraindikationen der Ergometrie zusammengefasst. Durchführungsempfehlungen werden dargestellt. Abbruchkriterien aufgrund pathologischer Auffälligkeiten oder bei Erreichen der Ausbelastung werden aufgelistet. Die Vor- und Nachteile der Stufenvs. der Rampenbelastung werden gezeigt. Neben den physiologisch zu erwartenden Reaktionen bei dieser Belastungsprüfung wird auch die eingeschränkte Sensitivität und Spezifität der Ergometrie für die Diagnostik einer koronaren Herzerkrankung am Beispiel der falsch-positiven und falsch-negativen Ergometrieuntersuchungen für Männer und Frauen im Alter von 50-59 Jahren mit unklaren Brustbeschwerden diskutiert. Die unterschiedlichen Ergometrieformen und ihre teilweise eingeschränkte Vergleichbarkeit untereinander und mit den reellen Arbeitsbedingungen erschweren darüber hinaus die Aussagekraft der Fahrradergometrie für die Beurteilung der körperlichen Leistungsfähigkeit eines Probanden, da lediglich die spezifisch fahrradergometrische Leistungsfähigkeit geprüft wird. Trotz den bei gesunden und jungen Probanden geringen Komplikationsraten sollte daher der Einsatz der Fahrradergometrie in der arbeitsmedizinischen Vorsorge kein Automatismus bei der Beurteilbarkeit der körperlichen Leistungsfähigkeit für den Einsatz des Probanden an einem bestimmten Arbeitsplatz sein.

\section{Schlüsselwörter}

Körperliche Leistungsfähigkeit · Koronare Herzerkrankung · Arbeitsplatz . Gefährdungsbeurteilung

\section{Bicycle ergometry in preventive occupational medicine precaution}

\section{Abstract}

A sufficiently high level of physical performance is of elementary importance for many occupational groups for coping with the daily work requirements. These include the fire brigade, police, military and also those employed in the construction industry, mining and agriculture. For the examination of the physical performance (bicycle) ergometry is available to the occupational physician as a standardized investigation, which is feasible under practical conditions.

Taking the national and international guidelines and current scientific literature into consideration, the knowledge, prerequisites, indications and contraindications of ergometry that are necessary for the occupational physician are summarized. The recommendations for implementation are presented. Abort criteria in cases of pathological abnormalities or when the maximum workload is reached are listed. The advantages and disadvantages of gradual vs. ramp loading are shown. In addition to the physiologically expected reactions in this stress test, the limited sensitivity and specificity of ergometry for the diagnostics of coronary heart disease are discussed using examples of false positive and false negative ergometry examinations for men and women aged $50-59$ years with unclear chest pains. Moreover, the different forms of ergometry and the partially limited comparability with each other and with real working conditions make the validity of bicycle ergometry for assessing the physical performance of a test person more difficult, since only the specific bicycle ergometric performance is tested. Therefore, despite the low complication rates in healthy and young volunteers, the use of bicycle ergometry in preventive occupational healthcare should not automatically be used in the assessment of physical performance for a subject's employment in a particular workplace.

\section{Keywords}

Physical fitness - Coronary heart disease . Workplace $\cdot$ Risk assessment konstant vorliegt, wird bei der rampenförmigen Belastung kontinuierlich nach Einstellung der Anfangslast (ebenfalls zumeist $50 \mathrm{~W}$ ) die Belastung gesteigert (• Abb. 1).

Um im Rahmen längerfristiger Betreuung sowohl intra- wie interindividuell vergleichbare Ergebnisse zu erzielen, sind standardisierte Untersuchungsprogramme zu nutzen. Ein Vorteil von Untersuchungsprogrammen mit kurzer Stufendauer und kleinen Leistungssprüngen bzw. von rampenförmigen Leistungssteigerungen besteht darin, dass hier relativ schnell eine kardiozirkulatorische Ausbelastung möglich ist, die in der Arbeitsmedizin noch verbreitet PWC („physical working capacity“) genannt wird $[10,12]$. Sie ist beim Rampentest leicht und nachvollziehbar $\mathrm{zu}$ berechnen. Ein möglicher Vergleich der Werte motiviert den Probanden, auch bei hohen Lasten weiterzutreten. Gleichzeitig kann bei einer Rampenbelastung durch größere Anstiegssteilheit (bei trainierten Männern z.B. $25 \mathrm{~W} / \mathrm{min}$ statt $25 \mathrm{~W}$ alle $2 \mathrm{~min}$ ) die Untersuchungsdauer reduziert werden [12]. Leistungsfähige
Probanden mit Maximalbelastung von weit über $300 \mathrm{~W}$ werden dennoch länger als oben empfohlen belastet.

Demgegenüber wird in der Stufenbelastung durch die Stufendauer eine konstante Belastung erreicht, wodurch leistungsdiagnostische Aussagen (u.a. mittels Laktatwerte) erst sinnvoll ermöglicht werden. 


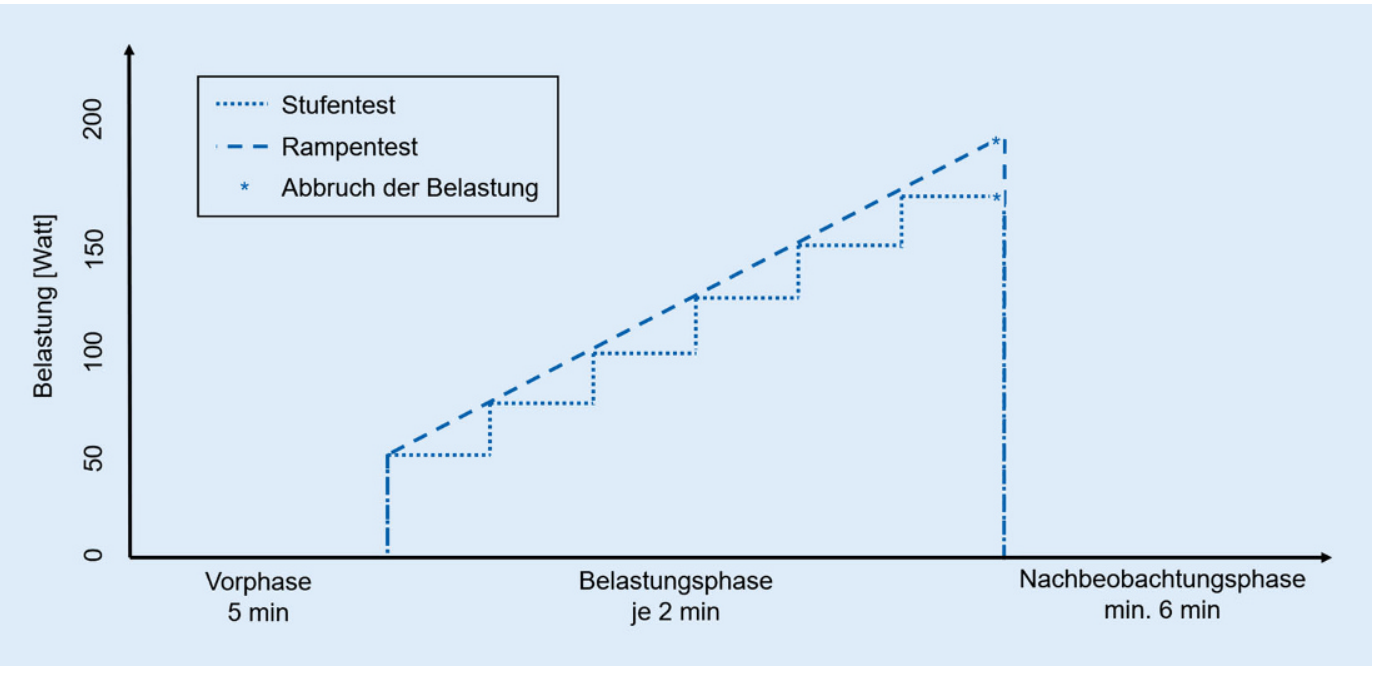

Abb. 1 Schematische Darstellung des Ablaufs einer Fahrradergometrie mittels Stufen- bzw. Rampenbelastung

\section{Verhalten kardialer, meta- bolischer und respiratorischer Parameter}

Während der Ergometrie kommt es während der Belastungssteigerung zur Erhöhung der Herzschlagfrequenz und der Herzauswurffraktion und damit zu einem Anstieg des Herzzeitvolumens, welches insbesondere in der Anfangsbelastung zunächst zu einer Ökonomisierung des Herzschlags führt. Gleiches trifft für die Atmung $\mathrm{zu}$, die mit zunehmender Belastung sowohl in Atemtiefe als auch Atemfrequenz zunimmt. Während der systolische Blutdruck mit zunehmender Belastung deutlich ansteigt, nimmt der diastolische Blutdruck lediglich leicht zu, kann aber auch konstant bleiben. Im Rahmen der Energiebereitstellung wird zunächst noch ausreichend Sauerstoff in der Muskulatur zur Verfügung gestellt (aerobe Phase), mit zunehmender Belastung kann das kardiopulmonale System nicht mehr ausreichend Sauerstoff zur Verfügung stellen, und es wird zunehmend die Energiebereitstellung in der Muskulatur unter Laktatproduktion gewährleistet (anaerobe Phase).

\section{Abbruchkriterien}

Die Ergometrie sollte bei Erreichen einer der in - Tab. 2 aufgezeigten Kriterien abgebrochen werden. Hierbei gilt analog zu den Kontraindikationen, dass bei arbeitsmedizinischen Untersuchun- gen der Maßstab der Abbruchkriterien eher strenger anzulegen ist.

Als Ausbelastungskriterium kann die maximale Herzschlagfrequenz ( $\left.\mathrm{Hf}_{\max }\right)$ mittels der Formel

$$
\mathrm{Hf}_{\max }=207-0,7 \times \text { Alter }
$$

ermittelt werden, bei deren Erreichen von einer Ausbelastung des Probanden ausgegangen werden kann $[13,14]$. Alternativ kann bei sportlich aktiven Probanden die im Training ermittelte $\mathrm{Hf}_{\text {max }}$ herangezogen werden. Die Ausbelastung des Probanden sollte bei der Überprüfung der körperlichen Leistungsfähigkeit angestrebt werden, sofern vorher keine anderen Kriterien zum Abbruch zwingen.

\section{Sensitivität und Spezifität zum Ausschluss einer koronaren Herzerkrankung}

Die Ergometrie wird neben der klinischen Beurteilung und zur Feststellung der körperlichen Leistungsfähigkeit zum Ausschluss einer koronaren Herzerkrankung (KHK) als ergänzende Untersuchung herangezogen [15]. Hierbei gilt es $\mathrm{zu}$ berücksichtigen, dass aufgrund der reduzierten Sensitivität und Spezifität (gewichtetes Mittel: $68 \%$ bzw. 77\% nach einer Metaanalyse basierend auf 147 Studien [16]) der diagnostische Stellenwert der Ergometrie im Vergleich zu anderen Testverfahren zur Diagnose einer KHK als Ursache von z.B. Brustbeschwerden eine geringere diagnostische Aussage- kraft besitzt. So wird in der Nationalen Versorgungsleitlinie (NVL) KHK [15] davon ausgegangen, dass bei einer hohen Vortestwahrscheinlichkeit ( $>30 \%$, siehe hierzu beispielhaft $\bullet$ Tab. 3) und einem negativen Belastungs-EKG die Nachtestwahrscheinlichkeit (falsch-negative Befunde in der Ergometrie) im Durchschnitt noch immer über $15 \%$ liegt, sodass weitere Tests zum Ausschluss der KHK erforderlich bleiben. Aufgrund der geringen Sensitivität und Spezifität führt jedoch die Ergometrie auch bei Probanden mit nichtanginösen Brustbeschwerden (geringe Vortestwahrscheinlichkeit) zu einer hohen Anzahl falsch-positiver Befunde (•Abb. 2) mit entsprechenden weitergehenden Untersuchungen, weshalb der Einsatz der Ergometrie zur singulären Vorsorge als KHK-Ausschluss (in der Arbeitsmedizin) nicht empfohlen werden kann. Der Betriebs- und Arbeitsmediziner sollte beim Verdacht auf eine KHK bei einem Probanden eine weiterführende Diagnostik beim zuständigen Hausarzt oder den entsprechend kardiologisch tätigen niedergelassen Internisten empfehlen. Weiterführende detaillierte Literatur hierzu findet sich bei Klingenheben und Kollegen [27].

Ferner ist zu berücksichtigen, dass bei der Einnahme bestimmter Medikamente bereits das Ruhe-EKG eines Probanden verändert ist. So führen DigitalisPräparate $\mathrm{zu}$ muldenförmigen ST-Senkungen bereits im Ruhe-EKG, wodurch die Aussagekraft der Ergometrie beeinträchtigt ist. Betablocker führen bekannt- 
Tab. 2 Abbruchkriterien von Belastungsuntersuchungen. (Mod. nach $[9,10])$

\begin{tabular}{|c|c|}
\hline & Abbruchkriterien \\
\hline \multirow{4}{*}{$\begin{array}{l}\text { Subjektive } \\
\text { Symptome }\end{array}$} & Erschöpfung des Patienten \\
\hline & Schwindel \\
\hline & Progrediente Angina pectoris \\
\hline & Progrediente Dyspnoe \\
\hline \multirow[t]{3}{*}{$\begin{array}{l}\text { EKG-Verände- } \\
\text { rungen }\end{array}$} & $\begin{array}{l}\text { Progrediente Arrhythmien: } \\
\text { Couplets, Salven (polymorphe Extrasystolie, Paare ( } 2 \text { konsekutive VES), Salven } \\
\text { [ } \geq 3 \text { konsekutive VES]), Kammertachykardien, zunehmende supraventrikuläre } \\
\text { Extrasystolen, Vorhoftachykardien, Vorhofflattern, neu auftretendes Vorhofflim- } \\
\text { mern, Bradyarrhythmien }\end{array}$ \\
\hline & $\begin{array}{l}\text { Progrediente Erregungsleitungsstörungen: } \\
\text { zunehmende QRS-Verbreiterung, Auftreten eines Linksschenkelblocks }\end{array}$ \\
\hline & $\begin{array}{l}\text { Progrediente Erregungsrückbildungsstörung: } \\
\text { ST-Senkung, horizontale Senkung über } 0,2 \mathrm{mV} \text {, progrediente ST-Hebung im } \\
\text { Elektrokardiogramm (EKG; >0,1 mV), monophasische Deformierung im EKG als } \\
\text { ST-Hebung (immer Abbruch) }\end{array}$ \\
\hline \multirow[t]{5}{*}{ Hämodynamik } & Progredienter Blutdruckabfall \\
\hline & Unzureichender Blutdruckanstieg (weniger als $10 \mathrm{~mm} \mathrm{Hg}$ pro Belastungsstufe) \\
\hline & $\begin{array}{l}\text { Übermäßiger Blutdruckanstieg (ca. } 250 \mathrm{~mm} \mathrm{Hg} \text { auf niedriger oder mittlerer } \\
\text { Belastungsstufe) bzw. hypertensive Fehlregulation (systolisch } 230-260 \mathrm{~mm} \mathrm{Hg} \text {, } \\
\text { diastolisch } \geq 115 \mathrm{~mm} \mathrm{Hg} \text { ) }\end{array}$ \\
\hline & Klinische Zeichen einer Unterversorgung mit Sauerstoff (Zyanose) \\
\hline & $\begin{array}{l}\text { Blutdruckabfall }>10 \mathrm{~mm} \mathrm{Hg} \text { (Vergleich zum Ausgangsblutdruck) mit Zeichen } \\
\text { einer myokardialen Ischämie (Angina pectoris, ST-Senkung) }\end{array}$ \\
\hline \multirow[t]{2}{*}{ Sonstiges } & Technische Probleme (defekte EKG-Registrierung, Monitorausfall) \\
\hline & Erreichen der Ausbelastung aufgrund der erreichten Herzschlagfrequenz \\
\hline
\end{tabular}

Tab. 3 Vortestwahrscheinlichkeit hinsichtlich signifikanter koronarer Herzerkrankung (KHK) bei symptomatischen Patienten in Abhängigkeit von Alter und Geschlecht. (Nach [17])

\begin{tabular}{|c|c|c|c|c|c|c|}
\hline \multirow[t]{2}{*}{$\begin{array}{l}\text { Alter } \\
\text { (Jahre) }\end{array}$} & \multicolumn{2}{|c|}{$\begin{array}{l}\text { Nichtanginöse } \\
\text { Brustbeschwerden }\end{array}$} & \multicolumn{2}{|c|}{$\begin{array}{l}\text { Atypische Angina } \\
\text { pectoris }\end{array}$} & \multicolumn{2}{|c|}{$\begin{array}{l}\text { Typische Angina } \\
\text { pectoris }\end{array}$} \\
\hline & Männer & Frauen & Männer & Frauen & Männer & Frauen \\
\hline $30-39$ & 4 & 2 & 34 & 12 & 76 & 26 \\
\hline $40-49$ & 13 & 3 & 51 & 22 & 87 & 55 \\
\hline $50-59$ & 20 & 7 & 65 & 31 & 93 & 73 \\
\hline $60-69$ & 27 & 14 & 72 & 51 & 94 & 86 \\
\hline
\end{tabular}

Die angegeben Werte entsprechen der Prozentzahl der untersuchten Patienten mit hämodynamisch bedeutsamen Koronarstenosen in der Koronarangiographie

Tab.4 Vergleich der Sauerstoffaufnahme für unterschiedliche Belastungsformen, als Referenzgröße dient die ansteigende Laufbandbelastung. (Mod. nach [18])

\section{Art der Belastung}

Laufband, ansteigend Sauerstoffaufnahme (\%)

Laufband, horizontal 100

Fahrradergometrie, sitzend

95-98

Fahrradergometrie, liegend

93-96

82-85

Fahrradergometrie, sitzend, mit einem Bein

$65-70$

Armkurbelarbeit

65-70

Schwimmergometer

85

Stufentest (Step-Test) lich zur Verlangsamung des Herzschlags und können eine Ausbelastung des Probanden verhindern.

\section{Vergleich unterschiedlicher Ergometriemethoden}

Grundsätzlich beschränkt sich die Ergometrie nicht alleine auf eine fahrradergometrische Belastungsüberprüfung. Verschiedene Belastungsformen lassen sich grundsätzlich nur eingeschränkt miteinander vergleichen, es besteht aber die Möglichkeit, die Sauerstoffaufnahme miteinander in Beziehung zu setzen (-Tab. 4). Dies löst jedoch nicht das grundsätzliche Dilemma der eingeschränkten Übertragbarkeit der Ergometrie auf reelle Arbeitsbedingungen (s. unten)

\section{Übertragbarkeit auf reelle Arbeitsbedingungen}

Jede Belastungsuntersuchung prüft die Reaktion des Körpers auf die jeweils getestete Belastung ab. Entsprechend wird mittels der Fahrradergometrie auch die fahrradergometrische körperliche Leistungsfähigkeit des Probanden getestet. Dies führt insbesondere bei Probanden, die viel Fahrrad fahren, zu einer Überschätzung der körperlichen Leistungsfähigkeit. Darüber hinaus gilt es insbesondere, zwischen der absoluten Ergometerleistung in Watt und der relativen Ergometerleistung in Watt pro Kilogramm Körpergewicht zu unterscheiden. Da im Rahmen der Fahrradergometrie der Einfluss des Körpergewichts reduziert wird, erzielen übergewichtige Probanden ein verhältnismäßig besseres Ergebnis [28]. Insgesamt ist die Übertragbarkeit der fahrradergometrischen körperlichen Leistungsfähigkeit auf die tatsächliche körperliche Leistungsfähigkeit am Arbeitsplatz eingeschränkt, was u. a. bei Feuerwehrleuten [19] und beim Vergleich der Fahrradergometrie mit einem militärischen Fitnesstest festgestellt wurde [20]. Bei Probanden, die unter leistungsdiagnostischer Indikation (Eignungsuntersuchung z.B. nach FwDV 7 [Atemschutz] oder 8 [Tauchen]) Schwierigkeiten mit der Fahrradergometrie haben, kann im Einzelfall auf 


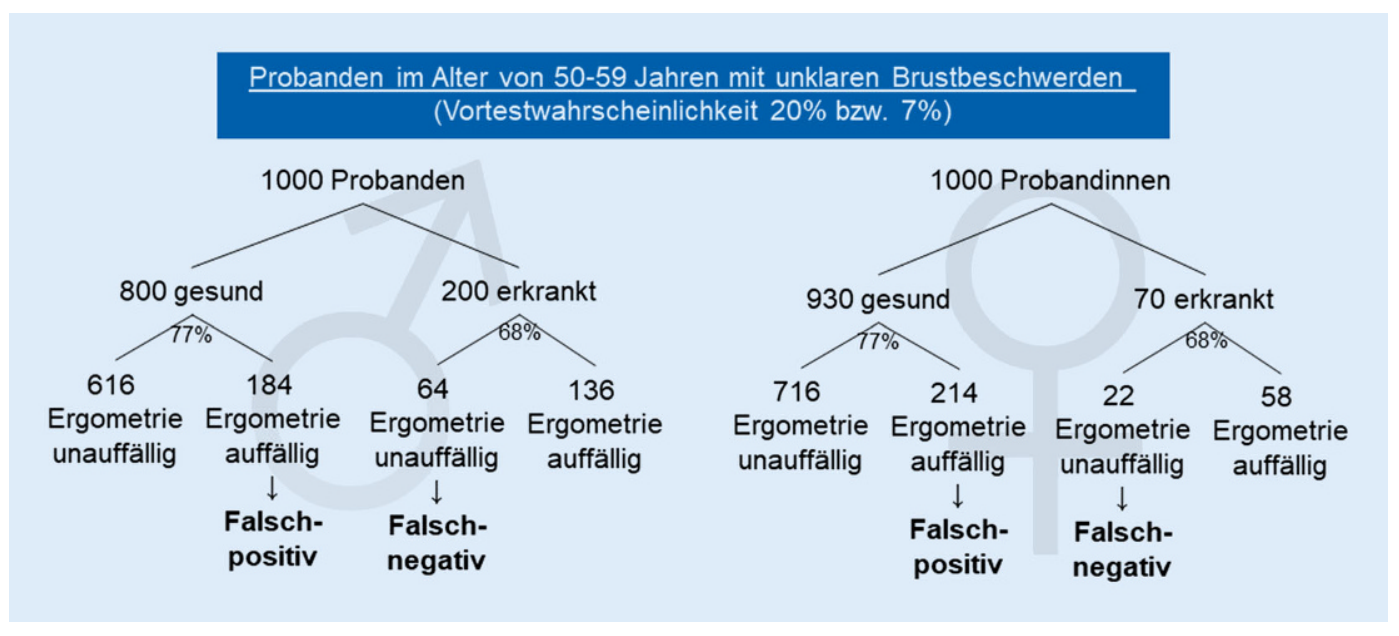

Abb. $2 \triangleleft$ Darstellung der falsch-positiven und falschnegativen Ergometrieuntersuchungen für Männer (links) und Frauen (rechts) im Alter von 50-59 Jahren mit unklaren Brustbeschwerden (Vortestwahrscheinlichkeit $20 \%$ bzw. $7 \%$ nach [17])

berufsspezifische Ergometrieformen wie die Leiter- oder Flossenergometrie zurückgegriffen werden [3,4]. Alternativ kann eine Spiroergometrie auf dem Fahrrad durchgeführt werden, wenn die Leistungsanforderungen im Beruf durch Messung der Sauerstoffaufnahme als Surrogatparameter z. B. mittels mobiler Spiroergometrie bestimmt worden sind $[21,22]$.

\section{Komplikationsrate}

Die Komplikationsrate für die Fahrradergometrie wird nach einer schon etwas älteren Publikation mit 18,4 Komplikationen auf 10.000 Untersuchungen angegeben [24]. Hierbei liegt die Rate der lebensbedrohlichen Komplikationen bei 1,5-5,2 pro 10.000 Untersuchungen und die des Todes der Probanden infolge der Durchführung der Ergometrie bei 0,2-0,4 auf 10.000 Untersuchungen $[23,24]$. Es gilt jedoch zu berücksichtigen, dass $82 \%$ der Komplikationen bei Patienten älter als 50 Jahre auftraten und $89 \%$ der Komplikationen bei Patienten auftraten, die zugleich eine Herzerkrankung aufwiesen. Die Komplikationsrate bei Sportlern und bei Probanden jünger als 20 Jahre liegt dagegen bei 0 [23, 24].

\section{Fazit für die Praxis}

- Die Fahrradergometrie kann - mit Einschränkungen - die körperliche Leistungsfähigkeit von Probanden ermitteln.

\section{- Die Übertragbarkeit auf die reellen Arbeitsbedingungen ist jedoch eingeschränkt. \\ - Dem gegenüber steht die standar- disierte und weit verbreitete Durch- führbarkeit dieser Messmethode. \\ - Die Fahrradergometrie besitzt bei der Detektion von kardiovaskulä- ren Erkrankungen (insbesondere KHK) nur eine sehr eingeschränkte Aussagekraft.}

\section{Korrespondenzadresse}

\section{PD Dr. S. Sammito}

Fachgruppe I 3 Foschung \& Erprobung,

Zentrum für Luft- und Raumfahrtmedizin der Luftwaffe

Flughafenstraße 1, 51147 Köln, Deutschland drsammito@web.de

Funding. Open Access funding provided by Projekt DEAL.

\section{Einhaltung ethischer Richtlinien}

Interessenkonflikt. S. Sammito, F. Heblich und N. Güttler geben an, dass kein Interessenkonflikt besteht.

Für diesen Beitrag wurden von den Autoren keine Studien an Menschen oder Tieren durchgeführt. Für die aufgeführten Studien gelten die jeweils dort angegebenen ethischen Richtlinien.

Open Access. Dieser Artikel wird unter der Creative Commons Namensnennung 4.0 International Lizenz veröffentlicht, welche die Nutzung, Vervielfältigung, Bearbeitung, Verbreitung und Wiedergabe in jeglichem Medium und Format erlaubt, sofern Sie den/die ursprünglichen Autor(en) und die Quelle ordnungsgemäß nennen, einen Link zur Creative Commons Lizenz beifügen und angeben, ob Änderungen vorgenommen wurden.

Die in diesem Artikel enthaltenen Bilder und sonstiges Drittmaterial unterliegen ebenfalls der genannten Creative Commons Lizenz, sofern sich aus der Abbildungslegende nichts anderes ergibt. Sofern das betreffende Material nicht unter der genannten Creative Commons Lizenz steht und die betreffende Handlung nicht nach gesetzlichen Vorschriften erlaubt ist, ist für die oben aufgeführten Weiterverwendungen des Materials die Einwilligung des jeweiligen Rechteinhabers einzuholen.

Weitere Details zur Lizenz entnehmen Sie bitte der Lizenzinformation auf http://creativecommons.org/ licenses/by/4.0/deed.de.

\section{Literatur}

1. Statistisches Bundesamt (2018) Statistisches Jahrbuch 2018. Statistisches Bundesamt, Wiesbaden, S353-384

2. Bundesministeriums der Justiz und für Verbraucherschutz (2019). Verordnung zur arbeitsmedizinischen Vorsorge vom 18. Dezember 2008 (BGBI. I S. 2768), die zuletzt durch Artikel 1 der Verordnung vom 12. Juli 2019 (BGBI.IS. 1082) geändert worden ist

3. Ausschuss Feuerwehrangelegenheiten, Katastrophenschutz und zivile Verteidigung (AFKzV) (2004). Feuerwehrdienstvorschrift 7 (FwDV 7, Aus gabe 2004) "Atemschutz". https://www.lfs-sh.de/ Content/Vorschriften/Dokumente/2004-08-00 \%20-\%20FwDV\%207\%20_Neufassung\%20SH_. pdf.Zugegriffen: 12. Aug. 2019

4. Ausschuss Feuerwehrangelegenheiten, Katastrophenschutz und zivile Verteidigung (AFKzV) (2014). Feuerwehr-Dienstvorschrift 8 (FwDV 8, Ausgabe 2014) „Tauchen". https://www.lfs-sh. de/Content/Vorschriften/Dokumente/FwDV_82014.pdf.Zugegriffen: 12. Aug. 2019

5. Fletcher GF, Ades PA, Kligfield P, Arena R, Balady GJ, Bittner VA, Coke LA, Fleg JL, Forman DE, Gerber TC, Gulati M, Madan K, Rhodes J, Thompson PD, Williams MA, American Heart Association Exercise Cardiac Rehabilitation, and Prevention Committee of the Council on Clinical Cardiology, Council on Nutrition, Physical Activity and Metabolism, Council on Cardiovascular and Stroke Nursing, 
and Council on Epidemiology and Prevention (2013) Exercise standards for testing and training. Circulation 128:873-934

6. Guazzi M, Arena R, Halle M, Piepoli MF, Myers J, Lavie CJ (2016) 2016 focused update: clinical recommendations for cardiopulmonary exercise testing data assessment in specific patient populations. Eur Heart J 133:1-18

7. Trappe HJ, Löllgen H (2000) Leitlinien zur Ergometrie. Z Kardiol 89:821-837

8. Wonisch M, Berent R, Klicpera M et al (2008) Praxisleitlinien Ergometrie. J Kardiol Austrian J Cardiol 15:3-17

9. Löllgen H, Erdmann E, Gitt A (Hrsg) (2010) Ergometrie - Belastungsuntersuchungen in Klink und Praxis, 3., vollständig überarbeitete Auflage. Aufl. Springer, Heidelberg

10. Löllgen $H$, Leyk D (2018) Exercise testing in sports medicine. Dtsch Arztebl Int 115:409-416

11. Bruce RA, Cooper MN, Gey GO, Fisher LD, Peterson DR (1973) Variations in responses to maximal exercise in health and in cardiovascular disease. Angiology 24:691-702

12. Heblich F, Gröbel H, Hauptmann M (2018) Criticizing German medical fitness tests for firefighters. Dtsch ZSportmed 69:5-12

13. Sammito S, Thielmann B, Seibt R, Klussmann A, Weippert M, Böckelmann I (2015) Guideline for the application of heart rate and heart rate variability in occupational medicine and occupational science. ASU International. https://doi.org/10.17147/ASUI. 2015-06-09-03

14. Tanaka H, Monahan KD, Seals DR (2001) Agepredicted maximal heart rate revisited. J Am Coll Cardiol 37:153-156

15. AWMF (2019) Nationale Versorgungs-Leitlinie Chronische KHK Langfassung, 5. Aufl. (Version 1 AWMF-Register-Nr.: nvl-004)

16. Gianrossi R, Detrano R, Mulvihill D, Lehmann $K$ Dubach P, Colombo A, McArthur D, Froelicher V (1989) Exercise-induced ST depression in the diagnosis of coronary artery disease. A metaanalysis. Circulation 80:87-98

17. Gibbons RJ, Chatterjee K, Daley J, Douglas JS, Fihn SD, Gardin JM, Grunwald MA, Levy D, Lytle BW, O'Rourke RA, Schafer WP, Williams SV Ritchie JL, Cheitlin MD, Eagle KA, Gardner TJ, Garson A Jr, Russell RO, Ryan TJ, Smith SC Jr (1999) ACC/AHA/ACP-ASIM guidelines for the management of patients with chronic stable angina: a report of the American College of Cardiology/American Heart Association Task Force on Practice Guidelines (Committee on Management of Patients With Chronic Stable Angina). J Am Coll Cardiol 33:2092-2197

18. Åstrand PO, Rodahl I, Dahl HA, Stomme SB (2003) Textbook of work physiology, 4. Aufl. Human Kinetics, Champaign

19. Heblich F, Sammito S (2015) Belastung von Atemschutzgeräteträgern - wie tauglich ist eine Tauglichkeitsuntersuchung. Zbl Arbeitsmed 65:87-91

20. Sammito S, Gundlach N, Böckelmann I (2016) Correlation between the results of three motor fitness tests (endurance, strength, speed) and the output measured during a bicycle stress test. Mil Med Res 3:12

21. Heblich F, Kähler W (2019) Erhöhte Belastung von Feuerwehrleuten durch die Atemschutzmaske? Zbl Arbeitsmed, online first https://doi.org/10. 1007/s40664-019-00367-5

22. Fröhlich MJ, Kroidl RF, Welte T (2018) Oxygen Consumption ( $\mathrm{V}^{\prime} \mathrm{O} 2$ ) and physical strain as measured by the occupational activity of cleaning personnel. J Occup Med Toxicol 13:4. https://doi. org/10.1186/s12995-018-0185-x

23. Kaltenbach M, Scherer D, Dowinsky S (1982) Complications of exercise testing - a survey in three German-speaking countries. Eur Heart J 3:199-202

24. Atterhög JH, Jonsson B, Samuelsson R (1979) Exercise testing: a prospective study of complications rates. American Heart Journal 98:572-579

25. Friedmann-Bette B (2011) Die Spiroergometrie in der sportmedizinischen Leistungsdiagnostik. Dtsch ZSportmed 62(1):10-15

26. Westhoff $M$, Rühle $K H$, Greiwing $A$, Schomaker $\mathrm{R}$, Eschenbacher $\mathrm{H}$, Siepmann $\mathrm{M}$, Lehnigk $B$ (1380) Ventilatorische und metabolische (Laktat-)Schwellen Positionspapier der Arbeitsgemeinschaft Spiroergometrie. Dtsch Med Wochenschr 2013:275-280

27. Klingenheben T, Löllgen $H$, Bosch R, Trappe HJ (2018) Manual zum Stellenwert der Ergometrie. Kardiologe 12:342-355

28. Kindermann W (1987) Recommendation for ergometry in medical practise. Dtsch Z Sportmed 38:244-268 